\title{
Challenges to the translation of basic science findings to atrial fibrillation therapies
}

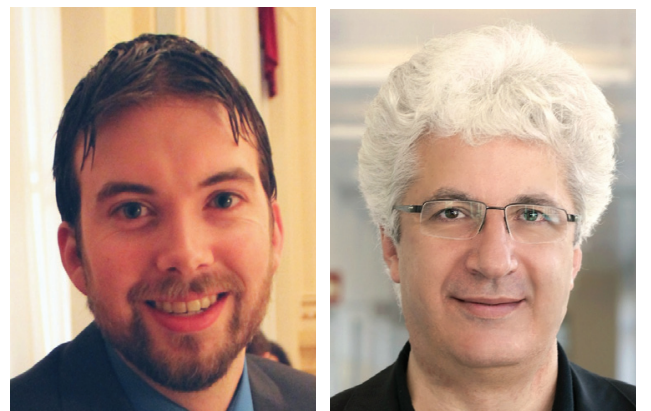

Jordi Heijman' \& Dobromir Dobrev*,2
"Although recent data suggest that ... atrial fibrillation therapy has improved in recent years, it remains suboptimal ... for both forms of rhythm control, which show limited efficacy and potentially severe side effects."

First draft submitted: 9 February 2016; Accepted for publication: 9 February 2016; Published online: 19 April 2016

Atrial fibrillation (AF) strongly impacts present day cardiovascular care, affecting an estimated 33.5 million people worldwide. AF increases morbidity and mortality, predominantly as a risk factor for stroke and worsening heart failure [1,2]. AF is considered a progressive disease and is typically classified as paroxysmal, persistent or longlasting persistent (chronic) based on the duration of episodes. The incidence of AF is expected to increase significantly in the upcoming years due to the ageing of the population, larger abundance of risk factors and improved detection, substantially increasing healthcare costs [1]. Current AF therapy involves antithrombotic therapy to reduce stroke risk and control of ventricular activation rate (rate control) [2]. In addition, cardioversion and subsequent sinus rhythm maintenance (rhythm control), predominantly through pharmacological approaches or ablation, are applied to symptomatic patients [2]. Although recent data suggest that overall AF therapy has improved in recent years, it remains suboptimal, particularly for both forms of rhythm control, which show limited efficacy and potentially severe side effects $[3,4]$.

There is a long-standing history of basic research in AF (reviewed in [1,5-6]). A better understanding of AF is expected to facilitate development of improved therapeutic approaches [6]. We have recently provided a critical analysis, highlighting the prominent role that basic insights have already played in current AF therapy, but also identifying a substantial translational gap [6]. Here, we will briefly summarize four important challenges in the translation of basic science findings to improved therapy and management and highlight recent discoveries that show promise for future improved mechanism-based AF therapy.

It remains challenging to identify 'multiscale' AF mechanisms

A detailed understanding of AF pathophysiology from molecule to patient is

\section{KEYWORDS}

- ablation - antiarrhythmic drugs

- arrhythmia mechanisms • atrial fibrillation • patient stratification - translation

\section{“Besides further refinements at each individual scale, future research linking microscopic mechanisms and macroscopic mapping will be key to further optimize atrial fibrillation therapy."}




\section{"A better classification of atrial fibrillation will help to translate specific atrial fibrillation mechanisms to the appropriate clinical cohort, likely improving antiarrhythmic efficacy in clinical trials."}

likely required for effective therapy. At present, most research has focused on the two extremes of this scale: cellular and molecular mechanisms as basis for novel antiarrhythmic drugs and in vivo imaging/mapping of $\mathrm{AF}$ manifestation to guide ablation.

It is obvious that the development of improved antiarrhythmic therapy requires detailed understanding of the molecular AF mechanisms to identify drugable targets. Since arrhythmias are an intrinsically multicellular phenomenon, the function of a single molecular target strongly depends on its environment and regulation in vivo. The molecular determinants of atrial arrhythmogenesis remain incompletely understood, but recent work has identified novel components that might represent potential therapeutic targets, including ion channels preferentially controlling atrial repolarization (e.g., two-poredomain $\mathrm{K}^{+}$-channels [7]), creating new opportunities to develop effective antiarrhythmic drugs with minimal ventricular side effects. Similarly, more information about signaling pathways regulating atrial electrophysiology and remodeling is becoming available. A prominent role for $\mathrm{Ca}^{2+} / \mathrm{CaMKII}$ in AF was validated in animal studies and samples from patients with chronic AF [8]. However, due to its diverse functions in various tissues, novel, isoform-specific CaMKII inhibitors modulating $\mathrm{Ca}^{2+} /$ calmodulin regulation or targeting of CaMKII to its protein substrates will be needed to validate the antiAF potential of CaMKII inhibition in clinical trials. Abnormal atrial metabolism and regulation of AMPK are also emerging contributors to the initiation and progression of AF [9], but their potential as antiarrhythmic targets requires further extensive investigation.

On the other hand, AF ablation was introduced without detailed understanding of molecular targets. However, optimizing the long-term success of ablation procedures necessitates insight into the structural and functional abnormalities promoting AF in an individual patient. Conceptually, AF can be maintained by repetitive ectopic activity and/or re-entry [1,5]. There has been a remarkable development in invasive and noninvasive imaging techniques to characterize the atrial substrate, resulting in procedures with improved long-term freedom from AF in some, but not all cohorts [3]. Nonetheless, it has proven challenging to definitively establish the mechanisms maintaining clinical AF [10] and consensus on the best ablation approach has not yet been reached. Evidence for both re-entrant activity and abnormal impulse formation exists, but the methodological differences between mapping approaches and the extensive signal processing makes the interpretation of mapping recordings challenging, and impede a direct connection to AF mechanisms [3,11-12].

Besides further refinements at each individual scale, future research linking microscopic mechanisms and macroscopic mapping will be key to further optimize AF therapy.

\section{- The etiology of AF patients is highly heterogeneous}

Although classification of AF as paroxysmal, persistent or long-standing persistent has important prognostic implications, for example, with respect to the success of AF ablation [3], it neglects the diverse etiology of AF patients. AF can occur in the presence of a wide range of risk factors and comorbidities [1], each of which may differentially modulate the proarrhythmic substrate and clinical consequences of AF. For example, some studies suggest that women have more thromboembolic complications and less benefit from AF therapy, resulting in a higher AF-related morbidity and mortality, indicating potential genderspecific mechanisms [13]. Similarly, improved cardiorespiratory fitness has recently emerged as a potential key element in AF therapy in the setting of obesity [14]. These data are in line with an increasing importance of epicardial fat in AF pathophysiology [15]. However, the exact molecular mechanisms and applicability to other patient cohorts are unclear.

A better classification of AF will help to translate specific AF mechanisms to the appropriate clinical cohort, likely improving antiarrhythmic efficacy in clinical trials. Cellular electrophysiological differences between atrial cardiomyocytes from patients with paroxysmal and chronic AF have provided information on distinct molecular mechanisms that precede the arrhythmia, versus those that result as a consequence of AF [5]. However, even for a given AF type, there is substantial patient-to-patient variability in the clinical presentation, ranging from numerous short-lived AF episodes to few but long-lasting episodes, and from completely asymptomatic to severely symptomatic patients $[1,5]$. Different clinical presentations likely reflect distinct AF mechanisms. This heterogeneity of AF makes translation of generic AF mechanisms to patient therapy challenging, although recent advances in the characterization 
of AF complexity may facilitate a more stratified approach. ECG biomarkers of AF complexity can predict long-term success of catheter ablation [16] and tailored therapy based on genetic information might also be possible and is currently being evaluated based on genetic variants near the PITX2 gene [17]. Nonetheless, extrapolating genetic alterations to AF mechanisms in patients remains challenging. For example, both gain and loss of PITX2 has recently been linked to increased AF risk, albeit through distinct mechanisms [18]. Moreover, there is very little information available about the influence of individual risk factors (e.g., heart failure or diabetes) on human atrial electrophysiology, calcium handling and related atrial remodeling.

\section{- Currently available preclinical models have important limitations}

Preclinical models play a key role in the development of new mechanistic insights and will be indispensable to obtain disease-specific stratification of AF therapy [5,6]. Animal models allow a better control of clinically relevant variables and comorbidities and enable multiscale studies by correlating in vivo and in vitro manifestations in the same animal. However, translation of findings from animal models to patients has proven challenging [19]. Among other things, most animal models do not show spontaneous AF initiation and subsequent progression, instead relying on burst pacing to assess substrate vulnerability. Similarly, the timecourse of disease is significantly shorter than in humans, likely affecting AF pathophysiology. Although human atrial samples reflecting the full disease complexity are available for preclinical investigations, they are generally limited to the right-atrial appendage and only available from patients undergoing open heart surgery, which is not representative for the majority of AF patients [5,6].

Emerging preclinical models, such as patientspecific induced pluripotent stem cell-derived cardiomyocytes and computational modeling might help to refine arrhythmogenic mechanisms by bridging the divide between species and scales [20], although such approaches also have limitations that need consideration $[6,20]$. A careful combination of experimental approaches is likely needed to establish fundamental AF-promoting mechanisms under well-defined conditions, develop tailored therapy for specific molecular targets and evaluate them in well-characterized clinical cohorts.

\section{- Limited understanding of structural} remodeling \& potential upstream therapy Current pharmacological approaches have mainly focused on inhibition of cardiomyocyte ion channels. Atrial structural remodeling, notably fibrosis, plays a major role in AF progression [21]. Increased atrial fibrosis is associated with increased duration of pacing-induced AF in animals, and slow heterogeneous conduction resulting from fibrosis is an established re-entry-promoting mechanism $[1,5,21]$. Although atrial fibrosis was associated with increased AF recurrence rate [21], overall fibrosis levels poorly correlate with AF complexity [22]. The molecular processes controlling fibroblast function, proliferation, differentiation and the bidirectional fibroblast-cardiomyocyte regulation remain unclear, limiting the success of upstream therapy aimed to prevent progressive AF-promoting remodeling. The latter strongly affects fibroblast proliferation and differentiation [23] and alters fibroblast ion channel function in AF patients $[24,25]$, but the physiological relevance and the pathways linking fibroblast ion channel function to fibrogenesis and eventually to cardiomyocyte dysfunction are largely unknown.

\section{Conclusion \& future perspective}

Technological and scientific innovations have greatly enhanced our understanding of AF mechanisms, which has already impacted clinical AF management. Nonetheless, many challenges remain. The diversity of clinical conditions and pathophysiological processes regulating AF likely preclude a 'one-size-fits-all' therapy. Thus, a close collaboration between basic scientists, cardiologists, cardiosurgeons, engineers, industry and regulatory authorities is needed to overcome the translational challenges and establish more tailored AF therapies. Recent developments are encouraging, suggesting that such approaches are indeed possible.

\section{Financial \& competing interests disclosure}

The authors' current work is supported by the German Federal Ministry of Education and Research through DZHK (German Center for Cardiovascular Research to D Dobrev) and The Netherlands Organization for Health Research and Development (ZonMW Veni 91616057 to J Heijman). The authors have no other relevant affliations or financial involvement with any organization or entity with a financial interest in or financial conflict with the subject matter or materials discussed in the manuscript apart from those disclosed.

No writing assistance was utilized in the production of this manuscript. 


\section{References}

1 Andrade J, Khairy P, Dobrev D, Nattel S. The clinical profile and pathophysiology of atrial fibrillation: relationships among clinical features, epidemiology, and mechanisms. Circ. Res. 114(9), 1453-1468 (2014).

2 January CT, Wann LS, Alpert JS et al. 2014 AHA/ACC/HRS guideline for the management of patients with atrial fibrillation: a report of the American College of Cardiology/American Heart Association Task Force on practice guidelines and the Heart Rhythm Society. Circulation 130(23), e199-e267 (2014).

3 Quintanilla JG, Perez-Villacastin J, Perez-Castellano N et al. Mechanistic approaches to detect, target, and ablate the drivers of atrial fibrillation. Circ. Arrhythm. Electrophysiol. 9(1), pii: e002481 (2016).

4 Ferrari R, Bertini M, Blomstrom-Lundqvist $\mathrm{C}$ et al. An update on atrial fibrillation in 2014: from pathophysiology to treatment. Int. J. Cardiol. 203, 22-29 (2016).

5 Heijman J, Voigt N, Nattel S, Dobrev D. Cellular and molecular electrophysiology of atrial fibrillation initiation, maintenance, and progression. Circ. Res. 114(9), 1483-1499 (2014).

6 Heijman J, Algalarrondo V, Voigt $\mathrm{N}$ et al. The value of basic research insights into atrial fibrillation mechanisms as a guide to therapeutic innovation: a critical analysis. Cardiovasc. Res. 109 (4) ), 467-479 (2016).

7 Schmidt C, Wiedmann F, Voigt $\mathrm{N}$ et al. Upregulation of $\mathrm{K}_{2 \mathrm{P}} 3.1 \mathrm{~K}^{+}$current causes action potential shortening in patients with chronic atrial fibrillation. Circulation 132(2), 82-92 (2015).

8 Heijman J, Voigt N, Wehrens XH, Dobrev D. Calcium dysregulation in atrial fibrillation: the role of CaMKII. Front. Pharmacol. 5, 30 (2014).
9 Harada M, Tadevosyan A, Qi X et al. Atrial fibrillation activates AMP-dependent protein kinase and its regulation of cellular calcium handling: potential role in metabolic adaptation and prevention of progression. J. Am. Coll. Cardiol. 66(1), 47-58 (2015).

10 Schotten U, Dobrev D, Platonov PG, Kottkamp H, Hindricks G. Current controversies in determining the main mechanisms of atrial fibrillation. J. Intern. Med. doi:10.1111/joim.12492 (2016) (Epub ahead of print).

11 De Bakker JM, Van Dessel PF. Long-standing persistent atrial fibrillation: can we distinguish ectopic activity from reentry by epicardial mapping? Circulation 132(22), 2103-2105 (2015).

12 Guillem MS, Climent AM, Rodrigo M, Fernandez-Aviles F, Atienza F, Berenfeld $O$. Presence and stability of rotors in atrial fibrillation: evidence and therapeutic implications. Cardiovasc. Res. 109 (4), 480 -492 (2016).

13 Tadros R, Ton AT, Fiset C, Nattel S. Sex differences in cardiac electrophysiology and clinical arrhythmias: epidemiology, therapeutics, and mechanisms. Can. J. Cardiol. 30(7), 783-792 (2014).

14 Pathak RK, Elliott A, Middeldorp ME et al. Impact of CARDIOrespiratory FITness on arrhythmia recurrence in obese individuals with atrial fibrillation: the CARDIO-FIT Study. J. Am. Coll. Cardiol. 66(9), 985-996 (2015).

15 Hatem SN, Redheuil A, Gandjbakhch E. Cardiac adipose tissue and atrial fibrillation: the perils of adiposity. Cardiovasc. Res. 109 (4), $502-509$ (2016).

16 Lankveld T, Zeemering S, Scherr D et al. Atrial fibrillation complexity parameters derived from surface ECGs Predict procedural outcome and long-term follow-up of stepwise catheter ablation for atrial fibrillation. Circ. Arrhythm. Electrophysiol. 9(2), e003354 (2016)
17 Darbar D. The role of pharmacogenetics in atrial fibrillation therapeutics: is personalized therapy in sight? J. Cardiovasc. Pharmacol. 67(1), 9-18 (2016).

18 Li N, Dobrev D, Wehrens XH. PITX2: a master regulator of cardiac channelopathy in atrial fibrillation? Cardiovasc. Res. 109(3), 345-347 (2016).

19 Nishida K, Michael G, Dobrev D, Nattel S. Animal models for atrial fibrillation: clinical insights and scientific opportunities. Europace 12(2), 160-172 (2010).

20 Heijman J, Erfanian Abdoust P, Voigt N, Nattel S, Dobrev D. Computational models of atrial cellular electrophysiology and calcium handling, and their role in atrial fibrillation. J. Physiol. 594(3), 537-553 (2016).

21 Dzeshka MS, Lip GY, Snezhitskiy V, Shantsila E. Cardiac fibrosis in patients with atrial fibrillation: mechanisms and clinical implications. J. Am. Coll. Cardiol. 66(8), 943-959 (2015).

22 Verheule S, Tuyls E, Gharaviri A et al. Loss of continuity in the thin epicardial layer because of endomysial fibrosis increases the complexity of atrial fibrillatory conduction. Circ. Arrhythm. Electrophysiol. 6(1), 202-211 (2013).

23 Harada M, Luo X, Qi XY et al. Transient receptor potential canonical-3 channeldependent fibroblast regulation in atrial fibrillation. Circulation 126(17), 2051-2064 (2012).

24 Du J, Xie J, Zhang Z et al. TRPM7-mediated $\mathrm{Ca}^{2+}$ signals confer fibrogenesis in human atrial fibrillation. Circ. Res. 106(5), 992-1003 (2010).

25 Poulet C, Kunzel S, Buttner E, Lindner D, Westermann D, Ravens U. Altered physiological functions and ion currents in atrial fibroblasts from patients with chronic atrial fibrillation. Physiol. Rep. 4(2), pii: e12681 (2016). 